\title{
Development of Handheld Haptics Device for Driving System of Unmanned Underwater Vehicles
}

\author{
Syed Mohamad Shazali ${ }^{1, *}$ \\ ${ }^{1}$ CeRIA, Faculty of Electrical Engineering, Universiti Teknikal Malaysia Melaka, 76100 Durian Tunggal, Malaysia
}

\begin{abstract}
This paper presents a research aimed at illustrating hydrodynamic force impact on the orientation of a Remotely Operated Underwater Vehicle (ROV) operating underwater by providing kinaesthetic haptic feedback to its handheld steering device. To get more understanding on how this aim can be achieved, a literature review had been done on the haptic feedback which are available to ROV pilots and how it could be delivered through a handheld device. While some achievement were made in providing different cues to pilots on drag force and its influence on its speed, non-have been made to offer insight on how it had affected ROVs orientation through haptic feedback. This study found that currently available handheld haptic device, while successfully delivering tactile feedback, are not capable of providing kinaesthetic feedback at par with the grounded haptic device. To address this, a series of thrusters has been introduced as a new actuation technique in providing kinaesthetic feedback on a handheld device in all three axes. This would allow total illustration of ROV orientation through haptic feedback. This paper has summarized and discussed our findings in our literature review, followed by some details of the proposed method.
\end{abstract}

\section{Introduction}

There is increasing demand for an unmanned underwater robot as the marine scientists and related industries are at high speed to grab the best the ocean has to offer and Remotely Operated Underwater Vehicle (ROV) seems to be the best options they have to do it. While undertaking tasks underwater, the vehicles do not enjoy the same luxury of sensors and communication capability like its counterpart on ground or in the sky, which usually presented as a visual cue to an operator. For these reasons, experienced operator had to rely only on visual information as prime feedback for most underwater robots operations [1], displaying information gathered from typically available sensor; depth sensors, compasses, side-scan and other sonars, thermistors, magnetometers and conductivity probes [2].

The operator's ability to comprehend ROV harsh underwater environment and driving the vehicle can badly affected by limited and fluctuating quality of visual feedback [3]. Research had been done to improve the visual feedback by embedding the vision feedback fed to the pilot with additional information on the environment underwater [4] [5]. Virtual Reality (VR) technology also had been used to increase the pilot capability to understand the current state and orientation of the ROV [6] [7]. However, the solution provided by this system had increases pilots' cognitive workload because of high reliance on the visual channel of feedback [8] while maintaining certain orientation in order to enable the vehicle to work. A report [9] indicate two main human factors issues regarding unmanned underwater vehicle; (a) vehicle control and monitoring display (b) perception of the underwater environment. This paper would highlight how our research addressing these issues by having haptic feedback in the system.

This research is about providing kinaesthetic haptic feedback on the orientation on ROV, illustrating hydrodynamic force impact (mainly drag and wave force) on the orientation, for a joystick-like handheld ROV steering device. This feedback is important to the pilot for at least two reasons; (1) it would be a great complement, if not as alternative, to visual feedback currently available to ROV pilots and (2) it would help the pilots to comprehend the impact of hydrodynamic force on the ROV orientation immediately and, in case of wave force, avoid it by diving deeper [10] or at least, in case of drag force, reduced it by reducing the speed or rate of motion of the vehicle.

Many works on haptic had been done, providing haptic feedback for handheld devices but most of them only produce the illusion of force vector [11] [12] [13] through tactile feedback and not a kinaesthetic force feedback which had been enjoyed by grounded haptic devices. This research would introduce the use of a series of thrusters to provide the force feedback in three axes and potential 6 degree of freedom (DOF) with a relatively low computational requirement.

The outlined of this paper is as follows: an overview of recent research on haptic feedback, with more attention

\footnotetext{
*Corresponding author: syedmohamad@utem.edu.my
} 
given to the handheld device, is presented in Section II. Section III covers our new proposed technique for illustrating ROV orientation and motion as well as the impact of hydrodynamic force on its orientation. Finally, we conclude our work and some hint of our future work in Section IV.

\section{Literature Review}

The motivation of this research is to provide haptic feedback on the orientation of an ROV, illustrating the impact of waves, water current and drag force on its orientation and motion. For this, a literature review exercise had been done to gain the understanding of how this forces affected the ROV orientation, what types of haptic feedback would be good for this purpose and currently available haptic feedback to ROV pilot. We also had explored on currently available haptic devices in order to find the best way to provide the required haptic feedback. In this section, the discussion on the impact of hydrodynamic force on ROV's orientation will be followed by the review of haptic feedback and how it could help addressing this issues.

\subsection{Wave and Drag Force on ROV orientation}

Although the magnitude of force exerted against an ROV, whether from the effects of water flow or water ripples caused by the vehicle or thruster nearby, is not a critical parameter to the operator, the resulting motion of the vehicle, which usually lead to changes in the position and orientation or speed of the vehicle, is very important in helping operators to react against it.

Many ROV operation need the operators to be able to tilt or pitch at certain roll or pitch angle frequently and steady enough to work, such as welding, or while driving the ROV at certain diving or pitching angle. This would be difficult as restoring moment, caused by buoyancy force and weight force would work against the motion that pitching or tilting the vehicle. While this phenomenon is very helpful as a passive stabilizing system to the ROV against the effect of wave and water current, some ROV designed to have a small restoring moment which allow the ROV to move and hovering at any tilting or pivoting angle with less effort needed.

A study [10] had shown that while the wave impact on ROVs on its roll and yaw moment would be less than $1 \mathrm{Nm}$, the impact on the vehicle pitch moment is very significant when fully submerge less than 15 metres from the free surface. This finding had resonant with previous research made on ROV motion in uniform current [14] where pitch angle significantly affected by the water current. Drag force, $F_{D}$, caused by fluid with $P$ density of the fluid and $C_{D}$ drag coefficient, on ROV with $A$ projected cross-sectional area moving at $u_{r}$ absolute speed, can be described as [3];

$$
F_{D}=\frac{1}{2} \rho u_{r}^{2} C_{D} A
$$

As the drag coefficient and cross-sectional area of the ROV would be constant and density of the fluid will be constant at a fixed temperature and pressure, drag force can be described with hydrodynamic coefficient, $K_{H}$, as

$$
F_{D}=u_{r}^{2} K_{H}
$$

with

$$
K_{H}=\frac{1}{2} \rho C_{D} A
$$

This shows that drag force would vary with the speed of the vehicle which depend on the motion caused by its thrusters and water current. Drag force does not directly affect the orientation of an ROV but rather oppose the motion involve while the vehicle changing its orientation, either cause by its own propeller or by its surrounding, including any external force acted on it.

\subsection{Haptic for ROV}

When we interact directly with objects in real life, the haptic sense is heavily involved in the perception of that interaction. It comprises two complementary senses; the tactile sense operates at the skin level, and allows feeling surfaces and textures and second, the proprioceptive or kinaesthetic sense, which is related to the perception of balance and posture of the different parts of the body, relative to each other [15]. Haptic technology recreates the sense of touch, benefitting from a combination of a somatosensory pattern of skin and proprioception of hand position, by applying forces, vibrations, or motions to the user [16].

The function of a haptic system is to provide reliable force feedback of the contact force at the slave side to the user manipulating a haptic device [17] while interacting with remote environments and allow a bi-directional flow of information [16]. While vision feedback dominantly used over the haptic feedback, it is very important when visual feedback is lost, unreliable or inadequate [18], although its limitations need to be tackled [19].

\subsection{Handheld Haptic Devices}

Haptic devices have a wide variety in workspace sizes, with most of these interfaces are grounded with limited workspaces [15]. Ungrounded haptic feedback devices offer a lot bigger workspace than grounded kinaesthetic devices, which use kinematic chains of rigid links and joints to transmit ground-based reaction forces to the user [20]. Handheld devices, which can take the form of gloves or exoskeleton device, can provide either kinaesthetic or tactile feedback to hands, especially the fingers, while not hindering the motions of the user like grounded devices. The leading approaches of providing haptic feedback on a handheld device had been summarized in Table 1. As many studies had been done on handheld haptic devices, simulating a lot of features of the surface texture, hardness and shape of an object, no research had been done to simulate the perception of net force applied onto an object.

While grounded haptic device enjoy the capability to produce kinaesthetic feedback through series of joints and 
linkages with reference to ground, handheld devices could only exploit the torque feedback using gyroscopic effect (GE) [21] and vibration as a tactile feedback [22] produced by various actuator, such as motors and thin film creating the haptic sensation of being twisted towards one direction the tablet. This approaches however limited to 1 DOF as the device has only one flywheel. Recently, visual feedback had been introduced to improve haptic feedback

Table 1. Leading Approach for Handheld Haptic Device

\begin{tabular}{|c|c|c|c|c|c|}
\hline Criteria & Force Feedback & Torque & \begin{tabular}{|l|} 
Tactile \\
Feedback \\
\end{tabular} & $\begin{array}{l}\text { Bimanual } \\
\text { Operation }\end{array}$ & Accuracy \\
\hline $\begin{array}{l}\text { Linear } \\
\text { Oscillatory } \\
\text { Actuator } \\
\text { (LOA) }\end{array}$ & $\begin{array}{l}\text { Linear Oscillatory } \\
\text { Actuator - pull, push, } \\
\text { and vertical } \\
\text { force/weight. [26] }\end{array}$ & No & $\begin{array}{l}\text { Simple } \\
\text { vibration on } \\
\text { horizontal plain }\end{array}$ & $\begin{array}{l}\text { Possible but no } \\
\text { specially design } \\
\text { device made for } \\
\text { bimanual } \\
\text { operation }\end{array}$ & $\begin{array}{l}\text { Great for the sense of direction } \\
\text { of the force, but not for an } \\
\text { accurate and sharp perception of } \\
\text { force direction. } 2 \text { DOF, } 2 \text { axes. }\end{array}$ \\
\hline $\begin{array}{l}\text { Gyro Effect } \\
(\mathrm{GE})\end{array}$ & No & $\begin{array}{l}\text { Directional } \\
\text { torque with } \\
\text { more than } 360^{\circ} \\
\text { workspace. } \\
{[21]}\end{array}$ & No & $\begin{array}{l}\text { Possible but } \\
\text { require some } \\
\text { modification on } \\
\text { the controller for } \\
\text { bimanual } \\
\text { operation. }\end{array}$ & $\begin{array}{l}\text { Great for directional torque with } \\
\text { an anchoring effect. }\end{array}$ \\
\hline $\begin{array}{l}\text { Electrical } \\
\text { Muscle } \\
\text { Stimulation } \\
\text { (EMS) }\end{array}$ & $\begin{array}{l}\text { Provide the illusion of } \\
\text { motion through } \\
\text { electrical stimulation } \\
\text { without really moving. } \\
\text { [32] }\end{array}$ & Not explored. & Possible & $\begin{array}{l}\text { Possible but not } \\
\text { tested for } \\
\text { bimanual } \\
\text { operation yet. }\end{array}$ & $\begin{array}{l}\text { No consistency and required } \\
\text { calibration for different users, } \\
\text { especially for force feedback } \\
\text { [31]. Application without vision } \\
\text { feedback had been reported to } \\
\text { be annoying/irritating. [13] } \\
\text { Better result achieved with } \\
\text { vision feedback. [28] }\end{array}$ \\
\hline $\begin{array}{l}\text { Skin Stretch } \\
\text { Feedback } \\
(\mathrm{SSF})\end{array}$ & $\begin{array}{l}\text { Varying speed and } \\
\text { magnitude of the tactor } \\
\text { motions to superimpose } \\
\text { force/ torque } \\
\text { movements could create } \\
\text { different tactile effects. }\end{array}$ & $\begin{array}{l}\text { Illusion of } \\
\text { torque }\end{array}$ & Yes & Yes. & $\begin{array}{l}\text { Great for a sense of direction of } \\
\text { the torque and inertia, but not } \\
\text { for an accurate and sharp } \\
\text { perception of horizontal force } \\
\text { direction. } 2 \text { DOF }\end{array}$ \\
\hline
\end{tabular}

[23].

All these feedbacks, however, did offer none or limited illustration of a force vector direction and cannot generate net forces on the limb, as required in providing kinaesthetic feedback, thus requiring the feedback, which usually only an illusion of force, to be processed and interpreted by the human before affecting physical behaviour change [20]. Handheld haptic device mostly approaches this issues by stimulating the skin, providing tactile or cutaneous feedback, using vibration [24] [11] or differential skin stretch feedback (SSF) providing both translating and angular force illusion to haptic user [12] to enable small, lightweight, and wearable or handheld devices. Although these approaches manage to provide a perception of force in a horizontal direction [25], this approach, however, failed to produce any perception of force in antigravity direction [24].

Linear oscillatory actuator (LOA) using an asymmetrical drive had been proposed as a haptic feedback [11], creating the illusion of being pulled and push as well as weight, achieved by a device based on 2 DOF oscillatory actuator which was significantly smaller and handy [26]. GE had been used to produce torque feedback. iTorqU, a handheld torque feedback device for haptic application [21] built to generate ungrounded torque feedback using a gimbal-controlled gyroscope. This research had been pursuit further with a gyroscopic actuator for TorqueScreen [27], an actuated flywheel imposing angular momentum for a handheld tablet, provided through electrical muscle stimulation (EMS) [28], motivated by the unsatisfactory haptic sense induced by EMS in the full spectrum of a haptic event.

GE did not display any translating force feedback and limited to only torque feedback in 2 axes, which is not available from LOA. SSF technique, however, manages to provide both torque and translating force sensation. EMS, on the other hand, had provided the illusion of motion through electrical stimulation but limited to the force caused by the motion of hand rather than the force from outside which apply onto an object. All of this approach, although had managed to provide good force and torque feedback, being ungrounded, none of this approach had provided any visual sense of orientation to the user which had been enjoyed by the grounded haptic devices' users.

Both SSF and LOA had the capability to provide tactile feedback with LOA produce a simple vibration on a horizontal plain. Beside GE devices, none of the above, as well as any of the approaches for handheld haptic device describe or mentioned before, had offered kinaesthetic force feedback and rely on illusion created with tactile feedback, which cannot be comparable enough to a grounded multi-DOF haptic device. It is also established that prolong vibration could cause perceptual fatigue [29]. All these lead to another option left to create sustained directional force for kinaesthetic feedback system, ejecting material. This has to be done safely and 
carefully controlled without annoying the pilot of such vehicle.

\section{The Handler}

A new handheld haptic device had been design to compliment a steering device which controlling orientation, instead of velocity, of an ROV. This design had, for the first time on a haptic device, a series of thrusters as its actuators. Each thruster comprised of a DC motor with propeller controlled through Pulse Width Modulation (PWM) signal. Acting as a steering device, orientation of the device will be captured through gyro sensor and feed as a reference orientation angle position for a driving system of an ROV. This device, The Handler (TH), had two systems; (1) ROV steering system and (2) haptic feedback system.

The former would have user desired ROV orientation as the input and ROV controller reference input as the output, while the later would have the ROV and the device orientation as the input and haptic feedback, both tactile and kinaesthetic feedback, as the output, as detailed in Table 2.

Table 2. The Handler's Input and Output Requirement

\begin{tabular}{|l|l|l|l|}
\hline Items & Requirements & Parameters & Resolution \\
\hline Inputs & Workspace & & \\
& Roll & $-90^{\circ}<\theta<90^{\circ}$ & \\
& Pitch & $-90^{\circ}<\theta<90^{\circ}$ & \\
& Yaw & $-90^{\circ}<\theta<90^{\circ}$ & \\
\cline { 2 - 4 } & Force & & \\
& Rotation & $<120 \mathrm{~N} . \mathrm{m},[10]$ & \\
\hline Outputs & Workspace & & \\
& Roll & $-90^{\circ}<\theta<90^{\circ}$ & \\
& Pitch & $-90^{\circ}<\theta<90^{\circ}$ & \\
& Yaw & $-90^{\circ}<\theta<90^{\circ}$ & \\
\cline { 2 - 4 } & Force & & \\
& Rotation & $0 \mathrm{~N} . \mathrm{m}<\mathrm{M}<11 \mathrm{~N} . \mathrm{m}$, & $2.25 \mathrm{~N}$ \\
& & {$[33]$} & $1 \mathrm{~N} . \mathrm{m}$ \\
\hline
\end{tabular}

This research had explored the opportunity to provide both tactile and kinaesthetic feedback. At its current stage, this device had been able to provide 20.6 N.mm for angular force rotating about $\mathrm{X}$ axis (roll) with a maximum vibration of $200 \mathrm{~Hz}$, as shows in Figure 1, and would have 5 thrusters for 3 axes haptic feedback for its final design. The device would control the direction and magnitude of its force feedback (FFB) in each axis with aim to provide resistance to the motion of the steering device. It would simulate different size or shape of ROV by varying the drag coefficient which then controls the amount of

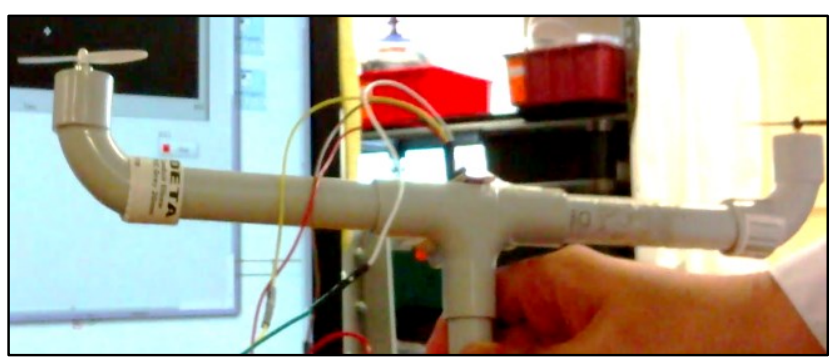

Figure 1. The Handler resistance provided by the device to the user. The device should be able to vary its magnitude and frequency of oscillating FFB according to the direction of the wave in respect of the ROV orientation.

\subsection{Drag force impact displays algorithm}

This research had developed an algorithm to display drag force on rotating ROV through a haptic device. While previous research had managed to capture the drag force in term of the ROV speed delay caused by the force, the approach was only applied to the drag force caused by a surge motion. As drag force exist when there is relative speed between the fluid and the surface of the vehicle, drag force will be captured by the difference between the angular orientation of the vehicle and $\mathrm{TH}$. The velocity vector, $V$, of an ROV is generally represented [30] as:

$$
V=\left[V_{1} V_{2}\right]^{T}=\left[\begin{array}{llllll}
u & v & w & p & q & r
\end{array}\right]^{T}
$$

with $u, v$ and $w$, are the component of the linear velocity vector, $V_{1}$, in the surge, sway and heave direction, while $p, q$, and $r$, are component of angular velocity, $V_{2}$, in the roll, pitch, and yaw. On the other hand, position vector, $\eta$, of an ROV is generally represented as:

$$
\eta=\left[\eta_{1} \eta_{2}\right]^{T}=\left[\begin{array}{lllll}
x & y & z & \varnothing & \theta
\end{array}\right]^{T}
$$

with $x, y$ and $z$, are the component of position vector, $\eta_{1}$, while $\phi, \theta$, and $\psi$, are orientation vector, $\eta_{2}$, for roll, pitch, and yaw. Utilizing simplified equation of drag force, the drag force of rotating ROV can be represented as:

$$
F_{D}=K_{H} V_{2}^{2}
$$

As hydrodynamic coefficient, $K_{H}$, would have different value for each side of the vehicle, $K_{H}$ could be represented as;

$$
K_{H}\left[\begin{array}{lll}
K_{H x} & K_{H y} & K_{H z}
\end{array}\right]^{T}
$$

with $\mathrm{K}_{\mathrm{Hx}}, \mathrm{K}_{\mathrm{Hy}}$ and $\mathrm{K}_{\mathrm{Hz}}$ are the hydrodynamic coefficients for each side facing $\mathrm{x}, \mathrm{y}$ and $\mathrm{z}$-axis. As haptic force, $F_{H}$, would have $F_{D}$ as the reference to provide the force feedback in each axis, with different gain $\left(K_{T H}\right)$, preset by user, for each axis, the haptic force could be described as

$F_{H}=K_{T H} F_{D}=K_{T H} K_{H} V_{2}^{2}=\left[\begin{array}{c}K_{T H x} K_{H x} p^{2} \\ K_{T H y} K_{H y} q^{2} \\ K_{Z}\left(\psi_{T H}-\psi_{R O V}\right)^{2}\end{array}\right]$

This equation had angular velocity, which is the difference of orientation in a certain of time. In providing the haptic feedback for The Handler, this equation had been modified to have differences between the device orientation, $\eta_{\mathrm{TH}}$, and the ROV orientation, $\eta_{\mathrm{ROV}}$, replacing the angular velocity in the equation. With

$$
q=\frac{\eta_{f}-\eta_{i}}{t_{f}-t_{i}}, t_{f}=t_{i}+1, \text { and } K_{n}=K_{T H n} K_{H n}
$$


The haptic force can be described with the different between the device orientation, $\eta_{\mathrm{TH}}$, and the ROV orientation, $\eta_{\mathrm{ROV}}$, as

\subsection{Actuator Control}

While the actual angular velocity is available, from an inertial measurement unit (IMU) that provides the gyro

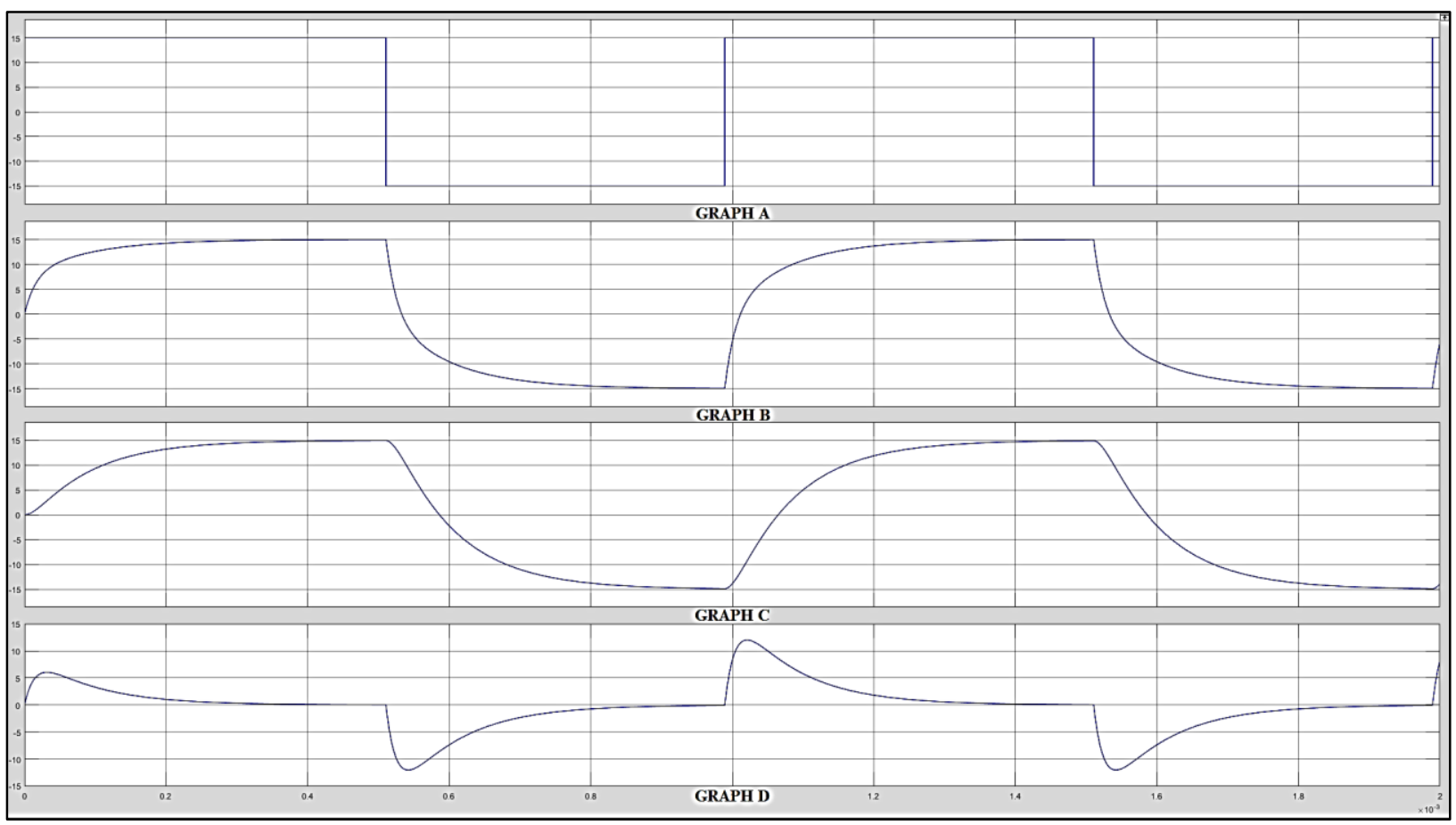

Figure 2. Simulation result illustrating the relation between the intended orientation angle and the haptic feedback produce by

$\mathrm{TH}$

$$
F_{H}=\left[\begin{array}{c}
K_{x}\left(\emptyset_{T H}-\emptyset_{R O V}\right)^{2} \\
K_{y}\left(\theta_{T H y}-\theta_{R O V y}\right)^{2} \\
K_{z}\left(\psi_{T H}-\psi_{R O V}\right)^{2}
\end{array}\right]
$$

As ROV, usually, do not have the uniform or cubic shape, each side would have different size of the surface area which then gives different drag coefficients for rotational motion about each axis. The algorithm should be able to display drag force impact on each surface with independently set drag coefficient. To do this, this device would capture the delay in the motion of the rotational motion and provide force feedback proportional to the amount of the delay, on the opposite direction of the motion.

To illustrate the outcome and behaviour of the haptic feedback which will experienced by the user, a simulation had been done by imposing a series of orientation input. Graph shown in Figure 2 shows the input of the driving system in the form of intended orientation in one axis. The user is expected to cause a little delay while moving the device, as shown in Graph B, which is represent the actual orientation of the device. Graph $\mathrm{C}$ represent the orientation of ROV which experienced significant delay caused by the drag force against it motion. The different between the orientation angle of ROV and TH had been captured in Graph D which will be the input for the Haptic System. sensor reading, this haptic device designed with an objective to provide information regarding the orientation of the vehicle, not drag force, to the user through the illusion of force similar to drag force. Since the force of the thrusters on the device would be controlled by varying voltage supply of its motors, the voltage supply, $V$, will be varied between its minimum and maximum to reflect the different between the device orientation, $\eta_{\mathrm{TH}}$, and the ROV orientation, $\eta_{\text {ROV }}$

$$
\begin{gathered}
V=k_{V}\left(\mathrm{\eta}_{T H}-\mathrm{\eta}_{R O V}\right) \\
k_{V}=\left(V_{H}-V_{L}\right) / 90
\end{gathered}
$$

where $V_{H}$ and $V_{L}$ are the maximum and minimum input voltage for the thrusters, which can be pre-set by the user, to control the magnitude of the kinaesthetic feedback.

\section{Conclusions and Future Directions}

This paper addressed force feedback for the haptic interface of ROV. Because of a direct measurement of applied force underwater is difficult, the haptic force vector had been derived from a proprioceptor sensor, the gyro sensor. The device had also offered both kinaesthetic and tactile feedback using its thrusters. However, the effectiveness of having both types of haptic feedback is yet to be investigated. 
On the later part of this research, the study will be made on the impact of kinaesthetic force feedback on handheld device output. Without a based, a handheld device would have the user's hand as the base of its relative motions. While the earth coordinate system would be the reference for the orientation of the device, a handheld device would not have the stiffness enjoyed by a grounded device. This would allow, depends on how the user reacts to the kinaesthetic feedback, significant influence by the actuator of the device on its orientation.

Unlike vibration, which can easily manage by most users, kinaesthetic feedback would be harder to handle. As this research aim at providing haptic feedback for a driving apparatus or device, this study would investigate how the haptic force vector pattern would be tolerated by the users and identify the limitation of the device without seriously affect the handheld device output, especially on the reference point for the ROV controller.

This work was performed under financial support from Universiti Teknikal Malaysia Melaka (UTeM) and The University of Auckland, New Zealand.

\section{References}

[1] J. Choi, Y. Lee, H.-T. Choi, J.-J. Kang and J.-H. Ryu, "A preliminary study on development of haptic interface for underwater vehicles," 2015.

[2] X. Lin and S. Guo, "Development of a spherical underwater robot equipped with multiple vectored waterjet-based thrusters," Journal of Intelligent \& Robotic Systems, vol. 67, no. 3, pp. 307-321, 2012.

[3] K. Le, H. Nguyen, D. Ranthumugala and A. Forrest, "Haptic driving system for surge motion control of underwater remotely operated vehicles," in Proceedings of the 6th International Conference on In Modelling, Identification \& Control (ICMIC), 2014.

[4] J. G. Sanchez, B. Patrao, L. Almeida, J. Perez, P. Menezes, J. Dias and P. Sanz, "Design and Evaluation of a Natural Interface for Remote Operation of Underwater Robots," IEEE computer graphics and applications, 2015.

[5] C. Domingues, M. Essabbah, N. Cheaib, S. Otmane and A. Dinis, "Human-Robot-Interfaces based on Mixed Reality for Underwater Robot Teleoperation," in 9th IFAC Conference on Manoeuvring and Control of Marine Craft (MCMC), 2012.

[6] M. Candeloro, E. Valle, M. R. Miyazaki, R. Skjetne and M. Ludvigsen, "HMD as a new tool for telepresence in underwater operations and closed-loop control of ROVs," in MTS/IEEE OCEANS 2015, Washington, 2015.

[7] A. Khadhraoui, L. Beji, S. Otmane and A. Abichou, "Stabilizing control and human scale simulation of a submarine ROV navigation," Ocean Engineering , vol. 114 , pp. 66-78, 2016.

[8] A. Vasilijevic, N. Miskovic and Z. Vukic, "Comparative assessment of human machine interfaces for ROV guidance with different levels of secondary visual workload," in 21st Mediterranean Conference on Control \& Automation (MED), 2013.

[9] G. Ho, N. J. Pavlovic, R. Arrabito and R. Abdalla, "Human Factors Issues When Operating Underwater
Remotely Operated Vehicles and Autonomous Underwater Vehicles (No. DRDC TORONTO-TM2011-100).," Defence Research and Development Toronto (Canada), Toronto, 2011.

[10] S. A. Malik, P. Guang and L. Yanan, "Numerical Simulations for the Prediction of Wave Forces on Underwater Vehicle using 3D Panel Method Code," Research Journal of Applied Sciences, Engineering and Technology, vol. 5, no. 21, pp. 5012-5021, 2013.

[11] T. Amemiya and T. Maeda, "Directional force sensation by asymmetric oscillation from a double-layer slidercrank mechanism," Journal of Computing and Information Science in Engineering, vol. 9 (1), pp. 1-8, 2009 .

[12] A. L. Guinan, M. N. Montandon, A. J. Doxon and W. Provancher, "Discrimination thresholds for communicating rotational inertia and torque using differential skin stretch feedback in virtual environments," in Haptics Symposium (HAPTICS) IEEE, 2014.

[13] F. Farbiz, Z. H. Yu, C. Manders and W. Ahmad, "An electrical muscle stimulation haptic feedback for mixed reality tennis game," 2007.

[14] M.-C. Fang, J.-H. Chen, J.-H. Luo and C.-S. Hou, "On the behavior of an underwater remotely operated vehicle in a uniform current," Marine Technology, vol. 45, no. 4, pp. 241-249, 2008.

[15] A. Talvas, "Bimanual haptic interaction with virtual environments," Doctoral dissertation, INSA de Rennes, 2014.

[16] S. Kapoor, P. Arora, V. Kapoor, M. Jayachandran and M. Tiwari, "Haptics - Touchfeedback Technology Widening the Horizon of Medicine," J Clin Diagn Res., 2014.

[17] T. Opitz and O. Meckel, "Control of Haptic Systems," in Engineering Haptic Devices, London, Springer , 2014, pp. 181-225.

[18] R. Balakrishnan and K. Hinckley, "The role of kinesthetic reference frames in two-handed input performance," Proc. ACM Symp. User Interface Softw. Technol., 1999.

[19] N. Gurari and G. Baud-Bovy, "Customization, control, and characterization of a commercial haptic device for high-fidelity rendering of weak forces," Journal of Neuroscience Methods, 2014.

[20] J. M. Walker, M. Raitor, A. Mallery, H. Culbertson, P. Stolka and A. M. Okamura, "A dual-flywheel ungrounded haptic feedback system provides single-axis moment pulses for clear direction signals," Philadelphia, USA, 2016.

[21] K. N. Winfree, J. Gewirtz, T. Mather, J. Fiene and K. J. Kuchenbecker, "A high fidelity ungrounded torque feedback device: The iTorqU 2.0.," in EuroHaptics conference, 2009 and Symposium on Haptic Interfaces for Virtual Environment and Teleoperator Systems. World Haptics 2009., 2009.

[22] G. Kato, Y. Kuroda, I. Nisky, K. Kiyokawa and H. Takemura, "HapSticks: A novel method to present vertical forces in tool-mediated interactions by a nongrounded rotation mechanism," 2015.

[23] S.-Y. Kim, J. Kim and K.-B. K. K. Kim, "A Film-type Vibrotactile Actuator for Hand-held Devices," The Journal of Korea Robotics Society, 2013. 
[24] T. Amemiya and T. Maeda, "Asymmetric Oscillation Distorts the Perceived Heaviness of Handheld Objects," IEEE Transactions on Haptics, vol. 1, no. 1, 2008.

[25] T. Amemiya, H. Ando and T. Maeda, "Lead-me interface for a pulling sensation from hand-held devices," $A C M$ Transactions on Applied Perception, vol. 5, no. 3, 2008.

[26] M. Kato, Y. Kono, K. Hirata and T. Yoshimoto, "Development of a Haptic Device Using a 2-DOF Linear Oscillatory Actuator," IEEE Transactions on Magnetics, vol. 50, no. 11, pp. 1-4, 2014.

[27] M. Murer, B. Maurer, H. Huber, I. Aslan and M. Tscheligi, "TorqueScreen: Actuated flywheels for ungrounded kinesthetic feedback in handheld devices," in TEI 2015 - Proceedings of the 9th International Conference on Tangible, Embedded, and Embodied Interaction, 2015.

[28] T. Ishikawa, T. Toshio and K. Yuichi, "Wearable Pseudo-Haptic Interaction by Using Electrical Muscle Stimulation," Haptic Interaction, pp. 135-140., 2015.

[29] S. Coren, Sensation and perception, John Wiley \& Sons, Inc., 2003.

[30] L. G. García-Valdovinos, T. Salgado-Jiménez, M. Bandala-Sánchez, L. Nava-Balanzar, R. HernándezAlvarado and J. A. Cruz-Ledesma, "Modelling, Design and Robust Control of a Remotely Operated Underwater Vehicle," International Journal of Advanced Robotic Systems , vol. 11, 2014.

[31] M. Pfeiffer, S. Schneegaß and F. Alt, "Supporting interaction in public space with electrical muscle stimulation," 2013.

[32] H. Kajimoto, "Illusion of motion induced by tendon electrical stimulation," 2013.

[33] V. M. Ciriello, R. V. Maikala and N. V. O’Brien., "Maximal acceptable torques of six highly repetitive hand-wrist motions for male industrial workers," Human Factors: The Journal of the Human Factors and Ergonomics Society, vol. 55, no. 2, pp. 309-322, 2013. 